\title{
Factors influencing exacerbation-related self-management in patients with COPD: a qualitative study
}

This article was published in the following Dove Press journal:

International Journal of COPD

28 November 2016

Number of times this article has been viewed

\author{
YJG Korpershoek ${ }^{1,2}$ \\ SCJM Vervoort ${ }^{3}$ \\ LIT Nijssen ${ }^{2}$ \\ JCA Trappenburg ${ }^{2}$ \\ MJ Schuurmans ${ }^{1,2}$
}

'Research Group Chronic Illnesses, Faculty of Health Care, University of Applied Sciences Utrecht, 'Julius Center for Health Sciences and Primary Care, University Medical Center Utrecht, ${ }^{3}$ Cancer Center, University Medical Center Utrecht, Utrecht, the Netherlands
Correspondence: YJG Korpershoek Research Group Chronic Illnesses, Faculty of Health Care, University of Applied Sciences Utrecht, Heidelberglaan 7, 3584 CS, Utrecht, PO Box I20|I-350| AA, Utrecht, the Netherlands

Tel +3I 638763949

Email yvonne.korpershoek@hu.nl
Background: In patients with COPD, self-management skills are important to reduce the impact of exacerbations. However, both detection and adequate response to exacerbations appear to be difficult for some patients. Little is known about the underlying process of exacerbationrelated self-management. Therefore, the objective of this study was to identify and explain the underlying process of exacerbation-related self-management behavior.

Methods: A qualitative study using semi-structured in-depth interviews was performed according to the grounded theory approach, following a cyclic process in which data collection and data analysis alternated. Fifteen patients (male $n=8$; age range 59-88 years) with mild to very severe COPD were recruited from primary and secondary care settings in the Netherlands, in 2015.

Results: Several patterns in exacerbation-related self-management behavior were identified, and a conceptual model describing factors influencing exacerbation-related self-management was developed. Acceptance, knowledge, experiences with exacerbations, perceived severity of symptoms and social support were important factors influencing exacerbation-related selfmanagement. Specific factors influencing recognition of exacerbations were heterogeneity of exacerbations and habituation to symptoms. Feelings of fear, perceived influence on exacerbation course, patient beliefs, ambivalence toward treatment, trust in health care providers and self-empowerment were identified as specific factors influencing self-management actions.

Conclusion: This study provided insight into factors influencing exacerbation-related selfmanagement behavior in COPD patients. The conceptual model can be used as a framework for health care professionals providing self-management support. In the development of future self-management interventions, factors influencing the process of exacerbation-related selfmanagement should be taken into account.

Keywords: COPD, self-management, exacerbation, qualitative research, grounded theory

\section{Introduction}

COPD is a highly prevalent chronic disease and a major cause of mortality worldwide. ${ }^{1}$ The natural course of COPD is interrupted by exacerbations characterized by a sustained worsening of patients' respiratory symptoms, which are beyond normal dayto-day variability and may warrant medical treatment. ${ }^{1-3}$ These exacerbations have a serious negative impact on the quality of life $\mathrm{f}^{4,5}$ and are associated with accelerated lung function decline and increased mortality. ${ }^{6,7}$ To address the burden of exacerbations, self-management has become increasingly important. ${ }^{8,9}$ Self-management skills are important to detect exacerbations early and to take prompt action to reduce the impact. ${ }^{10}$ However, both detecting exacerbations and taking prompt action appear to be difficult for patients. ${ }^{11-13}$ Previous studies have shown that approximately $50 \%$ of 
exacerbations are not reported to a health care professional and subsequently do not receive adequate treatment. ${ }^{10,14,15}$

Supporting patients in early detection of an exacerbation and teaching self-management skills using a written action plan have been shown to accelerate recovery time and decrease the acute impact of exacerbations on health status. However, no effects on the quality of life, exacerbation frequency and health care utilization have been observed. ${ }^{16}$ This may be explained by the sole focus on exacerbation onset and the "one size fits all" approach regarding the design, intensity and mode of delivery in different types of patients. ${ }^{17}$ Individualized and tailored strategies aimed at changing behavior across the full spectrum of exacerbation-related self-management are expected to result in a higher effect size. ${ }^{17,18}$ To be able to develop self-management interventions that are acceptable, valued by and effective in patients, more in-depth knowledge is needed on how patients perceive these events and behave accordingly. ${ }^{19}$

Previous research has shown that patients' perceptions toward exacerbations are complex and diverse. ${ }^{20-22} \mathrm{~A}$ recent meta-synthesis of qualitative studies has provided an improved understanding of patients' responses and appraisals of exacerbations. ${ }^{23}$ Furthermore, a recent study investigated patients' assessment and management of exacerbations at home and showed that they identified exacerbations by visible and invisible symptoms and that health care contact usually occurred when patients no longer felt able to manage their symptoms by themselves..$^{24}$ Although considerable research has provided insight into patients' perceptions and experiences regarding exacerbations, the underlying process of exacerbation-related self-management behavior is relatively unexplored. This lack of knowledge subsequently hampers the development of future exacerbation-related self-management interventions with higher effect sizes that fit patients' needs and requests for care. The objective of this study was to identify and explain the underlying process of exacerbation-related self-management behavior in COPD patients necessary for the development of future targeted and tailored self-management interventions.

\section{Methods}

\section{Study design}

A qualitative study was performed according to the principles of the grounded theory approach to generate a theory to explain COPD patients' exacerbation-related self-management behavior. ${ }^{25,26}$ The study was approved by the Medical Ethics Research Committee of the University Medical Center Utrecht (15-134/C).

\section{Sample and setting}

Initially, a purposive sample of Dutch patients with a clinical diagnosis of COPD, who had experienced at least one exacerbation in the past 12 months, was selected by health care providers (HCPs) from four general practices, one physiotherapy practice and one hospital in or near the city of Utrecht during the period of March-October 2015. Patients were eligible to participate if they met the inclusion and exclusion criteria described in Table 1. To increase the likelihood of reflecting different perspectives in the findings and ensuring representativeness, maximum variation sampling was used to create a large diversity in patients' age, sex, COPD severity and time since the last exacerbation. After the initial sampling, the data collection and analysis were alternated following a cyclic process, and theoretical sampling was used to select patients who could enrich the theory as it emerged. Theoretical sampling was continued as much as possible until saturation was reached. ${ }^{26}$

\section{Recruitment and informed consent}

Patients were informed about the study by their HCP. If a patient was willing to participate, patient's permission was asked to share contact details with the researcher. Subsequently, the patient received written study information and was contacted by the researcher to make an appointment for the interview. Written informed consent was obtained from all patients. Patients provided consent to consult their medical charts to obtain additional information on several predetermined characteristics. Twenty patients were approached for this study. In total, fifteen patients agreed to participate.

Table I Inclusion and exclusion criteria

\begin{tabular}{|c|c|}
\hline \multicolumn{2}{|c|}{ Patients with a clinical diagnosis of COPD } \\
\hline Inclusion & Exclusion \\
\hline Age $>40$ years & $\begin{array}{l}\text { Diagnosed with cognitive } \\
\text { impairments }\end{array}$ \\
\hline $\mathrm{FEV}_{\mathrm{I}} / \mathrm{FVC}$ ratio $<70 \%$ & $\begin{array}{l}\text { Primary diagnosis of asthma, } \\
\text { cardiac disease or other major } \\
\text { functionally limiting diseases }\end{array}$ \\
\hline $\begin{array}{l}\text { GOLD stage } \geq 2 \text {, spirometry } \\
\mathrm{FEV}_{1}<80 \% \text { predicted } \\
\text { Adequate communication skills }\end{array}$ & Life expectancy $\leq 3$ months \\
\hline$\geq \mathrm{I}$ reported exacerbation* in the & \\
\hline past 12 months prior to entering this & \\
\hline $\begin{array}{l}\text { study (to ensure adequate recall of } \\
\text { their experience of an exacerbation) }\end{array}$ & \\
\hline
\end{tabular}

Notes: *An exacerbation was defined as a period of symptom deterioration in which use of a course of corticosteroids and/or antibiotics was required or hospitalization was necessary. Data from Global Initiative for Chronic Obstructive Lung Disease (GOLD).'

Abbreviations: GOLD, Global Initiative for Chronic Obstructive Lung Disease; $\mathrm{FEV}_{\text {}}$, forced expiratory volume in I second; FVC, forced vital capacity. 
Four patients declined to participate, and one patient could not participate due to hospitalization.

\section{Data collection}

Semi-structured in-depth individual interviews were performed to investigate patients' perspectives toward exacerbation-related self-management. Each patient was interviewed once. A topic list was used as a framework for formulating open questions. The topics included perceived symptoms of COPD, perceptions toward exacerbations, performed actions, perceptions toward one's own role in exacerbation management and needs regarding self-management (Table S1). To clarify the meaning of an exacerbation, patients were asked specifically if they could remember a period of symptom deterioration for which a course of prednisolone and/ or antibiotics had been prescribed (Table S1). The topic list was adapted during the process based on emerging theoretical ideas. All interviews started with the same opening question: "How do you experience having a lung disease in your daily life?".

The interviews were conducted by a nurse scientist with a nursing degree (YK) and health scientist in training (LN). An expert on qualitative research with a nursing background was involved in the process of data collection to ensure methodological quality (SV). The interviews were held in patients' homes $(n=13)$ or at the hospital $(n=2)$. In three interviews, a partner was present. The duration of the interviews ranged from 45 to 90 minutes. The interview focus shifted to specific topics that were identified through the data analysis. In thirteen interviews, both researchers (YK and LN) were present, and they alternated between the interviewer and observer roles. All interviews were audiotaped. During and directly after the interviews, memos were created to describe observations, reflect on methodological issues and capture initial thoughts related to theoretical concepts.

Baseline characteristics were collected after the interviews through questionnaires. In addition, medical chart reviews were performed to obtain information on the recorded time period since the last exacerbation, lung function and comorbidities. Patient data were encoded and analyzed anonymously.

\section{Data analysis}

Data were analyzed according to the grounded theory approach by two independent researchers (YK and LN) and were discussed with a third researcher (SV). All interviews were transcribed verbatim. Data analysis was supported by NVivo 10.0 software (QSR International Pty Ltd,
Version 10, 2012). The analysis took place in a cyclic process through open coding, axial coding and selective coding and was alternated with data collection. ${ }^{26,27}$ Using constant comparison, the identified themes were reviewed for differences and similarities in interview fragments. ${ }^{26,28}$ Memo writing supported the process of analysis.

First, two researchers (YK and LN) read an interview in its entirety to get an overall picture and then summarized the interview by reflecting on the interview course and information obtained related to the research objective. Second, the interview was reread in more detail, and meaningful paragraphs were open coded independently by both researchers and discussed afterward to reach consensus. Using open coding, the interview data were segmented and an initial list of codes emerged. After five interviews, the analysis shifted toward axial coding, in which codes were categorized according to similarity and organized under main themes. ${ }^{26,27}$ After axial coding, new data were collected to support initial ideas on connections between categories. Analytic induction was performed after the analysis of ten interviews by testing initial theoretical ideas in new cases. Finally, through selective coding, the categories were refined and connections between the categories were integrated to identify the process of exacerbation-related self-management behavior, leading to a conceptual model explaining this process. Connections between categories were confirmed with the last two interviews leading to saturation. ${ }^{26}$ The third researcher's (SV) role in this process included coding a selection of interview data and participating in discussions on the data analysis.

The credibility of the study was enhanced by emphasizing the aim to learn from patients and the researchers' non-judgmental approach during the interviews. The potential for bias was diminished through the transcription of interviews and the use of researcher triangulation in all phases of the study. ${ }^{29}$ To further enhance the credibility of the study, the process of data analysis and interpretation was systematically discussed in our research team, comprising experts in the fields of nursing science, qualitative research, self-management and COPD (MS, SV, JT). ${ }^{29}$ In addition, an external expert on qualitative research performed a peer review on our methodological quality, and a clinical nurse specialist was consulted for a peer review on the interpretation of our conceptual model.

\section{Results}

Baseline characteristics of the study population are presented in Table 2. Maximum variation was achieved for disease 
Table 2 Baseline characteristics of the study population $(\mathrm{N}=15)$

\begin{tabular}{|c|c|c|c|c|c|c|c|c|}
\hline ID & Sex & $\mathrm{Age}^{\mathrm{a}}$ & $\begin{array}{l}\text { Living } \\
\text { situation }^{\mathrm{b}}\end{array}$ & $\begin{array}{l}\text { Education } \\
\text { level }^{c}\end{array}$ & Smoking & $\begin{array}{l}\text { GOLD } \\
\text { stage }^{\text {d }}\end{array}$ & $\begin{array}{l}\text { Self-reported } \\
\text { exacerbations per yeare }\end{array}$ & $\begin{array}{l}\text { Time period from last } \\
\text { exacerbation in months }\end{array}$ \\
\hline ROI & $F$ & 66 & A & Medium & Former & I & I & $1-3$ \\
\hline R02 & $M$ & 59 & LPC & Medium & Current & 3 & 3 & $1-3$ \\
\hline R03 & $\mathrm{F}$ & 64 & A & Medium & Current & I & I & $>12^{*}$ \\
\hline R04 & $\mathrm{F}$ & 74 & LP & Low & Former & 2 & 0 & $6-12$ \\
\hline R05 & $F$ & 74 & LP & Medium & Current & 3 & 1 & $\mathrm{I}-3$ \\
\hline R06 & $M$ & 73 & A & Low & Current & 2 & 3 & $6-12$ \\
\hline R07 & $\mathrm{F}$ & 81 & A & Medium & Former & 3 & 2 & $<1$ \\
\hline R08 & $F$ & 74 & A & High & Current & 2 & I & $\mathrm{I}-3$ \\
\hline R09 & $M$ & 67 & LP & Low & Former & 2 & 3 & $<\mathrm{I}$ \\
\hline RIO & $M$ & 88 & A & Medium & Former & 3 & 1 & $<1$ \\
\hline RII & $M$ & 76 & LP & Low & Former & 4 & 5 & $<1$ \\
\hline $\mathrm{R} / 2$ & M & 64 & LP & Medium & Current & 3 & 2 & $<1$ \\
\hline$R / 3$ & $\mathrm{~F}$ & 59 & LP & Low & Former & 2 & 5 & $I-3$ \\
\hline $\mathrm{R} / 4$ & $M$ & 64 & LC & High & Former & 3 & 1 & $6-12$ \\
\hline RI5 & M & 68 & LP & High & Former & 3 & 4 & $<1$ \\
\hline
\end{tabular}

Notes: ${ }^{a}$ Age at time of interview. ${ }^{b} \mathrm{~A}$, living alone; LP, living with life partner; LPC, living with life partner and children; LC, living without a partner and with children. 'Low, primary school through vocational training; medium, secondary school or vocational training; high, college or university degree. ${ }^{\mathrm{d} A c c o r d i n g}$ to GOLD classification in medical chart. ${ }^{e}$ Amount of exacerbations determined by amount of prescriptions of prednisone and/or antibiotics for worsening of lung symptoms, estimated by patients themselves. * Last exacerbation was 16 months ago.

Abbreviation: GOLD, Global Initiative for Chronic Obstructive Lung Disease.

severity (mild to very severe COPD), age (range 59-88 years), sex (almost equally distributed) and time since the last exacerbation ( $<1-12$ months). Prescriptions for self-medication (antibiotics and/or prednisolone) were lacking in the study population, except some patients who received prescriptions during the holidays.

\section{Patient perceptions toward exacerbations}

When answering our first question, almost all patients started talking about their daily symptoms and functional limitations. Most experienced symptoms were breathlessness, sputum production and fatigue. Hence, symptoms varied widely per individual. Functional limitations were mostly related to exercising, walking and talking. With the exception of two patients, all patients were familiar with the name COPD for their disease.

When asking patients about their experiences with periods of symptom deterioration for which medical treatment was indicated, two patients could not remember having such a period in the past year. Most patients had a clear memory of this period and perceived it as a very unpleasant experience sometimes invoking feelings of fear and anxiety. Only four patients were familiar with the term "exacerbation" or "lung attack" as terms to describe these periods of symptom deterioration. Other patients related these periods to pneumonia, infection or the flu. A few patients did not refer to a certain term since their HCP, in their opinion, had not explicitly mentioned a certain term. Several patients believed that some internal factors, such as emotions, stress and pushing the boundaries regarding activities, could influence exacerbation onset. Other patients attributed exacerbation onset to external factors, such as seasonal influences and air pollution.

\section{Patterns in patients' exacerbation-related self-management behavior}

Based on patients' stories, a conceptual model describing factors influencing exacerbation-related self-management was developed (Figure 1). Our conceptual model presents the underlying process of exacerbation-related self-management behavior by explaining its influencing factors. We identified several patterns in exacerbation-related behavior based on two important self-management skills: recognition of an exacerbation and performance of self-management actions. The results are presented by first explaining these patterns in patients' behavior followed by a description of factors that influence these patterns. The patterns in recognition serve as a starting point. With regard to recognition, three different patterns in patients were identified and detailed in our conceptual model: 1) early recognition, 2) late recognition and 3 ) difficulties with recognition. Subsequently, these patients showed different types of selfmanagement actions, which were subdivided into "absence of self-management actions", "self-management actions to reduce symptoms", and "contacting an $\mathrm{HCP}$ ", as explained in Table 3. 


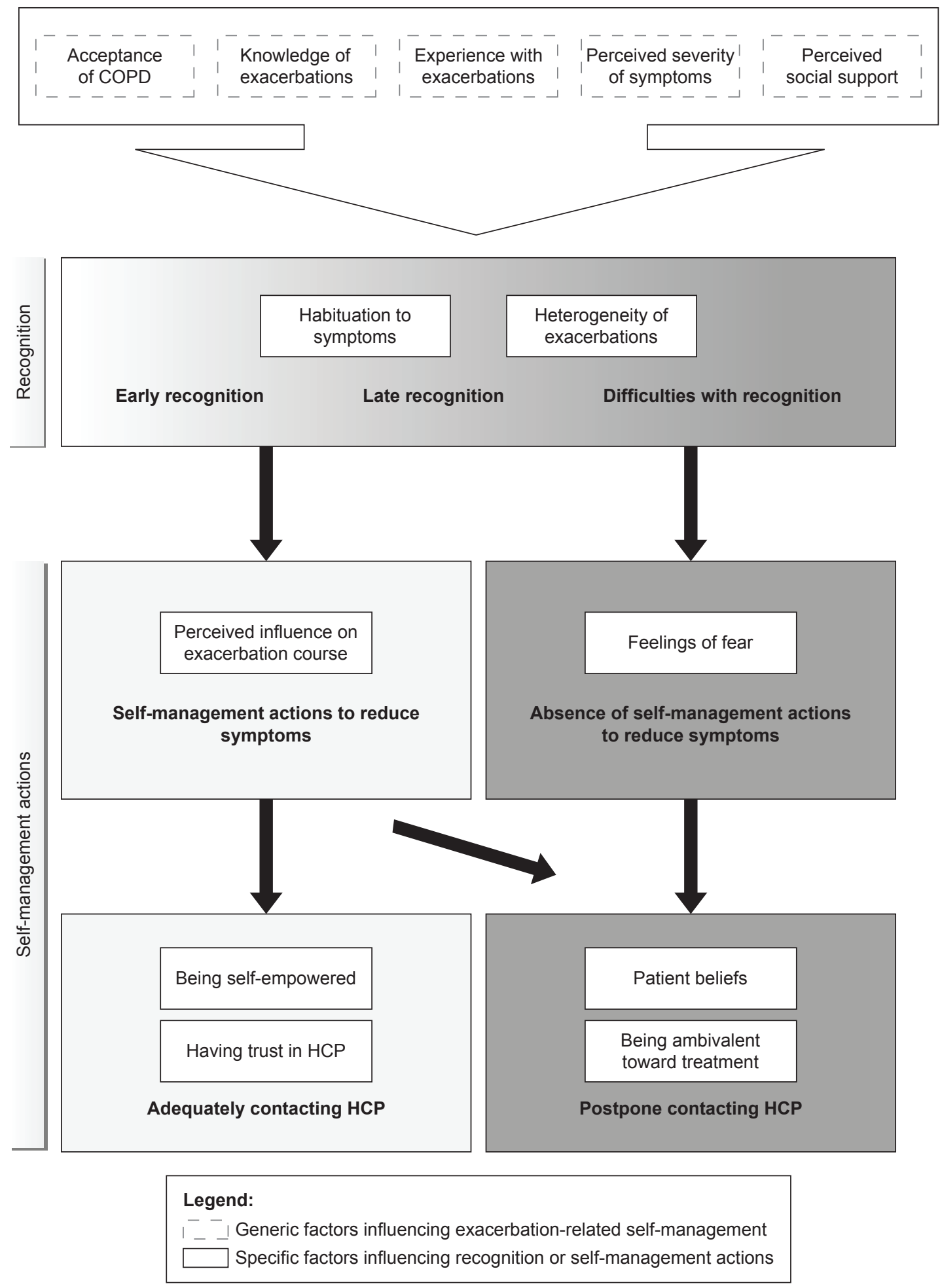

Figure I Conceptual model describing factors influencing recognition of exacerbations and performance of self-management actions. Abbreviation: $\mathrm{HCP}$, health care provider.

\section{Patients early recognizing exacerbations}

In general, patients who recognized an exacerbation often had experienced more than one exacerbation and perceived these events as a part of their COPD. Symptoms perceived as the onset of an exacerbation were increased fatigue, increased respiratory symptoms (eg, coughing, sputum production and breathlessness), specific pain and fever.

Patients who explained that they were able to recognize an exacerbation at an early stage were generally aware of the importance of early detection and prompt action. They had 
Table 3 Operationalization types of self-management actions

\begin{tabular}{|c|c|}
\hline Type of self-management actions & Explanation \\
\hline $\begin{array}{l}\text { Absence of self-management actions } \\
\text { to reduce symptoms }\end{array}$ & $\begin{array}{l}\text { Performed actions were similar to self-management actions in the stable phase (not experiencing an } \\
\text { exacerbation). Increasing inhalation medication was sometimes performed as patients are used to do } \\
\text { when experiencing daily fluctuations in symptoms. }\end{array}$ \\
\hline $\begin{array}{l}\text { Self-management actions to reduce } \\
\text { symptoms }\end{array}$ & $\begin{array}{l}\text { Taking rest, performing breathing or sputum expectoration techniques and increasing inhalation } \\
\text { medication/using an extra dosage of short-acting inhalation medication. If applicable: self-treatment } \\
\text { with antibiotics and/or prednisolone. }\end{array}$ \\
\hline Contacting an HCP & $\begin{array}{l}\text { - Adequately contacting HCP: Patients directly contacted an HCP in case of symptom deterioration } \\
\text { or monitored symptoms carefully and contacted an HCP when crossing the mutual agreed } \\
\text { threshold in symptoms. } \\
\text { - Postpone contacting HCP: Patients postponed to contact an HCP or mentioned that their HCP } \\
\text { advised them to contact earlier in the future. }\end{array}$ \\
\hline
\end{tabular}

Abbreviation: HCP, health care provider.

a pro-active attitude. By closely monitoring symptoms, they felt able to anticipate possible problems. R12: "I try to anticipate on that moment ... because I don't want to let things get that far that I will get a pneumonia". These patients often performed adequate self-management actions to reduce their symptoms and consulted their HCP on time. Some of these patients monitored their symptoms for 2 or 3 days when they felt able to manage the symptoms by themselves to ensure that the symptoms would not diminish on their own without prescription of medication. If their situation did not improve during this time, they contacted their HCP, or they contacted their HCP immediately in cases of sudden symptom increases. Some of these patients expressed a desire for self-treatment at home since they believed that this arrangement would allow them to take faster action. However, some patients who recognized exacerbations early postponed contacting their HCP.

\section{Patients recognizing exacerbations late}

Several patients recognized an exacerbation, but the recognition occurred late, when symptoms had already significantly increased over a number of days. These patients explained having difficulties interpreting their symptoms in a timely manner. Some of these patients realized afterward that symptoms had already been present before they recognized them. R11: "Well, afterwards you can say ... oh ... that was already present for a few days". Most of these patients continued their normal daily activities and performed various actions to minimize their symptoms. Several patients adequately contacted their HCP, although most patients postponed seeking medical help until they felt a strong urgency to do so. As a result, some patients reached a point of crisis leading to hospitalization. R02: "I often kept on going way too long. And then, at a certain moment, your blood is out of oxygen.
The last time in the hospital I only had 78\% oxygen in my blood. So I kept on going way too long”.

\section{Patients having difficulties recognizing exacerbations}

Patients having difficulties recognizing an exacerbation did not remember having an exacerbation or remembered receiving antibiotics and/or prednisolone for a temporary increase in symptoms but associated this period with the flu or with other problems dominating during that specific period. These patients did not seem to relate their temporary illness to their COPD. R05: “... and then my husband called the doctor. I said that is not necessary. It will resolve by itself. Everyone experiences flu sometimes". These patients generally performed little self-management actions. Their self-management actions were similar to their behavior in the stable phase, such as taking short-acting inhalation medication as they were used to doing. Overall, these patients postponed contacting an HCP and followed a wait-and-see approach. These patients expressed having little needs with regard to self-management support.

\section{Factors influencing exacerbation-related self-management behavior}

Figure 1 shows that we identified five generic factors influencing both recognition of exacerbations and performance of self-management actions either positively or negatively. In addition, specific factors influencing the ability to recognize an exacerbation or influencing the performance of self-management actions were identified. It is important to note that these factors mutually influenced each other as well. Details of these influencing factors are described in the following paragraphs and further illustrated by quotes in Table 4 ( $Q$ references in the text refer to specific quotes in Table 4). 
Table 4 Illustrative quotes related to identified factors influencing exacerbation-related self-management

Influencing factor
Acceptance of
COPD
Perceived severity of
symptoms

symptoms

Knowledge of exacerbations Experience with exacerbations

Perceived social support

Heterogeneity of exacerbations Habituation to symptoms

Perceived influence on exacerbation course Feelings of fear Being selfempowered

Having trust in HCP

Patient beliefs

\section{Quotes}

QI: "You can put on the brave face and say 'I got nothing' but that is nonsense of course. (...) You can better say 'it is this way'. Okay. And then you can react on it yourself” (R08).

Q2: "I mean. I have accepted that. I know that at some point it's not going to resolve so eh ehm ... In the beginning I would have had eh ehm ... a difficult time with it, but at the point where you accept it isn't going to go away, those lungs have been affected, the only thing possible is for it to be stable, that's my starting point" (RI4).

Q3: "I am someone who doesn't want to admit it at all, so you just keep doing everything” (R02).

Q4: "Yeah, then you start to wheeze a bit, but apart from that it it is not too bad" (RI0). (I: "yes ... but so ... you say it's not too bad ... but is it really alright then?") RI0: "Well ... not always ...".

Q5: “I don't think it bothers me that much. (...) My children say it does, but they look at it differently" (R08).

Q6: “I have seen it with my dad, he didn't go outside anymore, he just sat on a chair, kept on going but didn't do anything. But well, that's looking back, I never thought that I would also be such a COPD patient. But it did happen to me and now I think I have to stay physically active ... If you would like to resist those infections every time" (RI2).

Q7: "The sooner I contact my general practitioner, the sooner the medication is successful and the sooner I get rid of it again" (RI2).

Q8: "But then the symptoms will not resolve themselves ... you learn quite quickly that you should call immediately and

eh (...) But at some point you learn that: ok, this is a cough so you have to watch out ..." (RI5).

Q9: "At some point it will not pass and l've learned that if I feel something is coming up, I will call the general practitioner immediately" (R02).

QI0: “I don't want to let it get that far that I will get a pneumonia ... a heavy pneumonia ... I've had that before, and I was seriously shocked by that. I think it had also been bothering me for a couple of days then, and I waited too long ... now I think, I should raise the alarm sooner" (RI2).

QII: "Mum, you are short of breath ... Oh am I? And then I'm wheezing without even noticing it ..." (R08) (I: "But so then perhaps they recognize it sooner than you?") R08: "Yes".

QI2: ("I: When you decide to call your HCP, what influences that decision? Does your environment influence that decision as well?”) “Well ... very little ... that's just eh ... purely my own feeling” (RI5).

QI3: (“I: Do you feel like there is a difference between a gradual or sudden onset?") "Both are also possible (...) Symptoms are so different, you can't put your finger on it. That's too bad right, such a shame ..." (RI3).

QI4: (Partner and patient speaking about perceived symptoms) Partner of RII: "He also turn bluish quickly (...) but I often don't even notice because l'm so used to seeing that (...) but yeah, you experience it from the beginning you know? And see things slowly getting worse. And then it's difficult ... I think that's also why you notice it less". RI I adds: "Because the onset is gradually" (...) (RII).

QI5: "Yeah, look, because you always have it ... you learn to live with it. And when it gets worse, you know you have to warn, but because you are so used to it, you learn to live with it and you do everything with it" (RI0).

QI6: (I: “And why do you think it is important to take action on time?") "Well ... I think it will heal faster or something ... If I act sooner on it" (R06).

QI7: (I: “And what do you do when you feel more breathless?”) “Well nothing. You can't do anything” (RII).

Q I8: “At some point it's mainly fear, and well, I start hyperventilating and then I can't solve it anymore” (R02). Q 19: "When I feel terrible and I call the general practitioner, like the last time that I felt a pneumonia was emerging, and the assistant tells me I can visit the general practitioner next week I say: I am sick know, I want to see the doctor right now" (R06).

Q20: "So when I go to the general practitioner I know exactly what I want and I eh ... try to direct the general practitioner in that direction. If there are plausible reasons not to do that that's fine too, but before I go into the room I know for myself what I would like to accomplish" (RI4).

Q21: "And the assistants were not always that nice to me, they said the doctor doesn't have time today. And then I said: 'Yes, but' ... 'No, no time today, come in on Friday'. But then I visited the doctor and he asked me why I didn't come in earlier? Well ..." (RI3).

Q22: “When something goes wrong I will call that lung nurse because the pulmonologist isn't always available, you don't get to talk to him on the phone directly, but she can quickly pass on the information so that communication is pretty good" (RI5).

Q23: "Actually, the most important thing is recognition, early recognition of a pneumonia and that someone listens to you at the moment you think something is wrong" (RI3). (I: "Is that important to you, to take action?") RI3: "Yes, absolutely". Q24: (A patient mentioned that his doctor told him a few times to contact earlier and we asked the patient why the doctor gave this advice in the patients opinion. The patient answered:) "so you can have it treated sooner ... and then maybe it will recover sooner as well ... but, I am a bit stubborn" (RI0).

Q25: "Pushing through is what I learned back in the days (...) It is all mentality, if you feel mentally well, the rest will follow, because you feel responsible for the things that need to happen" (RI0).

Q26: “I am someone who thinks 'then my doctor is wasting time for nothing', so I wait until I am sick to death" (RI3). Q27: "That is how we were raised. Back in the day, you could not be sick (...) It is very hard to break that habit when you were raised like that (...)" (R08). 
Table 4 (Continued)

\begin{tabular}{|c|c|}
\hline Influencing factors & Quotes \\
\hline \multirow[t]{3}{*}{$\begin{array}{l}\text { Being ambivalent } \\
\text { toward treatment }\end{array}$} & $\begin{array}{l}\text { Q28: "You are not looking forward to going to the hospital. Although I actually know in advance that it will happen } \\
\text { anyway" (RII). }\end{array}$ \\
\hline & Q29: "Well, that you think: I would not call yet. I will wait a while (...) Prednisolone is an unpleasant medicine (...) \\
\hline & I would rather take nothing but well ... it is a necessary evil I say" (RI5). \\
\hline
\end{tabular}

Abbreviations: $\mathrm{HCP}$, health care provider; R, respondent; I, Interviewer; Q, quote.

\section{Generic factors influencing exacerbation- related self-management (both recognition and self-management actions) Acceptance of COPD}

Acceptance of COPD was identified as an important factor influencing patients' perceptions toward COPD and exacerbations, which subsequently affected exacerbation-related self-management. A wide variety of acceptance was found ranging from difficulties with acceptance to full acceptance. Patients who accepted their COPD perceived exacerbations as events they have to deal with and were therefore more likely to recognize an exacerbation early and to adapt their behavior to their current health status by taking prompt actions (Q1 and Q2). The level of acceptance varied in patients showing late recognition. Some patients acknowledged their symptom deterioration but neglected it and were unable to anticipate on these symptoms as they solely wanted to continue on with their normal lives (Q3). Patients having difficulties with acceptance preferred to avoid confronting their disease and often tried to hide it from others; in some cases, they did so because they felt ashamed. Some of these patients did not attribute an increase in symptoms to their COPD and therefore had difficulties with recognizing an exacerbation and taking prompt actions.

\section{Perceived severity of symptoms}

The perceived severity of symptoms varied widely from hardly noticing any symptom deterioration to taking symptom deterioration seriously. Patients who were aware of the gravity of their situation, perceiving exacerbations as hazardous events, often recognized exacerbations early. Furthermore, they explained that their situation would worsen if they postponed their actions. Perceived severity of symptoms varied in patients showing late recognition or difficulties with recognition. Some of them trivialized their situation by describing their symptoms as less serious than they actually were (Q4 and Q5). These patients often recognized an increase in symptoms but took a risk in postponing adequate self-management actions. To interpret their own severity, a substantial group of patients compared themselves to other patients with more severe COPD. These comparisons could influence patients' perceptions toward the severity of their symptoms in two different ways. On one hand, some patients trivialized their current condition by comparing themselves to others and therefore saw no reason to adapt their behaviors. On the other hand, the comparison led to patients' recognition of the gravity of their situation and created awareness about the progressive development of COPD, resulting in stronger intentions to perform adequate exacerbation-related self-management (Q6).

\section{Knowledge of exacerbations}

Patients with adequate knowledge of exacerbations often recognized exacerbations early and took prompt actions. Patients who were aware of the importance of taking prompt action were convinced that doing so could help them reduce exacerbation severity and recovery time (Q7). However, some of these patients postponed taking action as other influencing factors overruled. Patients having difficulties with recognition had little understanding of exacerbations and explained having little insight into actions they could perform to feel better.

\section{Experience with exacerbations}

Patients explained that their former experiences with exacerbations led to their increased confidence in recognizing future exacerbations, which was initiated by having a feeling when something is wrong or by recognizing a pattern of symptoms indicating that an exacerbation is imminent (Q8). Furthermore, patients who had experienced benefits from their past actions were more likely to perform adequate self-management actions (Q9). Negative experiences contributed to the learning curve as well. For example, patients who experienced hospitalization due to postponing their actions often recognized their boundaries better. Moreover, previously experienced feelings of fear and anxiety positively influenced patients' intentions to perform self-management actions, as they had learned to try to prevent situations that might invoke these feelings by contacting their $\mathrm{HCP}$ earlier (Q10). 


\section{Perceived social support}

Several patients expressed the importance of social support from family and personal relationships, as well as support from HCPs, in self-managing exacerbations. The influence of social support seemed to be highest for patients having difficulties recognizing exacerbations, as they often needed support from family or personal relationships to realize that symptoms were worsening and to stimulate health care contact (Q11). Social support seemed to be less important for patients who recognized exacerbations, although these patients sometimes needed others' support as well to take symptoms seriously and to take prompt actions. Patients who recognized exacerbations early and took prompt action often felt that they were able to manage exacerbations themselves (Q12). However, when these patients nonetheless postponed contacting an $\mathrm{HCP}$, social support was an influencing factor in the decision to contact an HCP as well.

\section{Factors influencing recognition of exacerbation}

\section{Heterogeneity of exacerbations}

Heterogeneity of exacerbations was identified as a key factor in distinguishing patients showing late recognition from patients showing early recognition. Some patients felt very capable of recognizing an increase in symptoms but perceived the heterogeneity of exacerbations as a barrier. These patients found it difficult to distinguish an exacerbation from normal day-to-day symptom variations, as the onset of an exacerbation could vary from gradual to sudden, and the types and severity of symptoms were not always consistent (Q13).

\section{Habituation to symptoms}

Recognition of an exacerbation was complicated by habituation to the progression of perceived daily symptoms. Patients who experienced habituation to symptoms explained that it became more difficult to determine a significant increase in symptoms, since they had learned to cope with the severity of their symptoms (Q14 and Q15).

\section{Factors influencing self-management actions}

\section{Perceived influence on exacerbation course}

Patients' perceived influence on the course of an exacerbation varied widely and was identified as a factor influencing self-management actions. Patients who felt able to influence the course of an exacerbation had a greater internal locus of control and were more likely to take self-management actions to reduce the severity of an exacerbation (Q16). In contrast, patients who felt that they could not influence the course of an exacerbation often postponed taking action (Q17).

\section{Feelings of fear and anxiety}

Some patients expressed feelings of fear and anxiety that limited their ability to perform adequate self-management actions to reduce symptoms. They stated that feelings of fear and anxiety were present at the time of acute symptom deterioration and included a fear of dying due to increased breathlessness and feelings of choking. These patients were often aware of strategies to reduce these perceived symptoms (such as breathing techniques and relaxation); yet, these feelings of fear and anxiety interfered with performing these strategies (Q18).

\section{Factors influencing contacting an HCP Being self-empowered}

Being self-empowered was identified as a factor that differentiated patients who contacted their HCP early from patients who postponed contact with their HCP. Self-empowerment refers to the extent to which patients are able to take action based on their own thoughts, believing that they know what is best for themselves. Self-empowered patients in the study prioritized their own health and were able to stand up for themselves (Q19 and Q20). Patients who postponed contact with their HCP were less self-empowered and mostly influenced by a threshold that they perceived. Some of these patients experienced organizational issues as a barrier to contacting their HCP, such as not getting an opportunity to talk with their HCP. These patients were not able to stand up for themselves, resulting in a delay of action with occasionally serious consequences (Q21).

\section{Having trust in $\mathrm{HCP}$}

Having trust in an HCP was identified as a factor that influenced patients contacting their $\mathrm{HCP}$ as patients described the importance of adequate coaching from their HCP in their selfmanagement actions. Patients who took prompt action often had agreements with their $\mathrm{HCP}$ on how to act in response to exacerbations and expressed having confidence in their $\mathrm{HCP}$ (Q22). Some patients learned what to do in case of symptom deterioration but felt that they were not taken seriously by their HCP when they felt a need to contact their HCP, leading to postponed health care contact. In addition, some patients experienced difficulties with explaining the seriousness of their symptoms to their HCP. Patients explained that being heard by an HCP contributed to their performance of selfmanagement actions (Q23). 


\section{Patient beliefs}

For a number of patients, the threshold for contacting an HCP was influenced by their beliefs. Some patients explained that they postponed contacting an HCP out of stubbornness despite knowing better (Q24). Some patients explained having hope that their symptoms would resolve themselves and therefore postponed contacting their HCP. Other patients believed that their social responsibilities (eg, housekeeping, work) were more important than listening to their own feelings (Q25). Finally, some patients explained that they did not want to bother their HCP and therefore postponed health care contact (Q26). A few people explained that this belief was influenced by how they were raised (Q27). Patients who adequately contacted their HCP were sometimes familiar with these beliefs, but having learned from their earlier negative experiences, they were able to adapt their beliefs and consider their own health to be most important.

\section{Being ambivalent toward treatment}

Many patients were ambivalent toward taking medication, especially with regard to prednisolone, due to its negative side effects. Consequently, some of these patients postponed contacting their $\mathrm{HCP}$ as they preferred not to admit that they needed treatment, although they were aware of its importance. Furthermore, ambivalence toward treatment was reflected by patients who tried to avoid hospitalization and therefore postponed contacting their HCP (Q28). Conversely, patients who were convinced that they needed treatment were more likely to contact their HCP (Q29).

\section{Discussion}

This study resulted in a conceptual model explaining factors influencing exacerbation-related self-management. Based on patients' stories, several patterns in exacerbation-related self-management were identified based on two important self-management skills: recognition of an exacerbation and performance of self-management actions. The identified patterns were dynamic in nature and could change in individual patients over time due to variability in influencing factors and disease progression. Our conceptual model shows that exacerbation-related self-management was influenced by acceptance of COPD, perceived severity of symptoms, knowledge of exacerbations, former experiences with exacerbations and social support. In addition, specific factors influencing the ability to recognize an exacerbation or the performance of self-management actions were identified.

Several of the findings of this study were in line with other studies. Corresponding with previous research, we found that patients often had difficulties understanding the term "exacerbation"22,24 and that patients reported both personal and environmental factors that may cause exacerbations. ${ }^{30}$ Our finding that exacerbation-related self-management behavior was determined by personal beliefs, perceptions regarding seriousness of the disease, knowledge of exacerbations and former experiences with exacerbations is consistent with the Health Belief Model. ${ }^{31-33}$ Furthermore, in line with previous research, acceptance was considered to be important for behavior change toward self-management, ${ }^{34,35}$ and the importance of social support was strengthened by previous research emphasizing the need to involve others in self-management support to change behavior. ${ }^{36}$ Consistent with previous research focusing on self-management actions, our study found that feelings of fear and anxiety resulted in perceived powerlessness in response to symptoms ${ }^{23}$ and perceived control on exacerbation course influenced selfmanagement actions. ${ }^{37-40}$ Previous studies have also observed differences between patients who pro-actively monitored the course of an exacerbation and contacted their HCP on time and patients who postponed health care contact until feeling an urgency for medical care. ${ }^{21,24,30,41}$ Finally, regarding smoking in relation to self-management, our study concurred with earlier studies in showing that smoking was not an explanatory factor for self-management behavior. ${ }^{42,43}$

Our findings expand upon prior work by identifying patient beliefs, ambivalence toward treatment, self-empowerment and trust in HCPs as specific factors influencing health care contact. Patient belief was identified as a barrier for seeking medical help since various patients felt that they "bothered" their HCP. As patients explicitly mentioned their upbringing as influencing factor, this finding might be due to this older population of COPD patients being raised not to bother other people with personal problems..$^{21,41}$ Furthermore, some patients who postponed health care contact did so because they felt that they were "not being heard" by their HCP. This finding might be explained by previous research showing that patients sometimes feel that they do not have legitimized reasons to seek help..$^{21,23}$ The identified ambivalence toward treatment can be explained by the Health Belief Model and by previous work showing that considering the side effects of medication was related to medication adherence..$^{24,31}$

To date, the literature has been inconclusive regarding patients' skills toward recognition of exacerbations. Williams et $\mathrm{al}^{24}$ showed that patients "just know" when they are experiencing exacerbations and that the majority of patients feel capable of distinguishing "bad days" from 
exacerbations, while our study identified patients showing early recognition, late recognition and patients having difficulties with recognition. These differences could be due to the recruitment strategies in the study of Williams et al. As it was part of a trial evaluating a self-management support intervention, the sample of patients may have had more engagement in self-management. ${ }^{24}$ However, in line with our study, Adams et $\mathrm{al}^{21}$ showed that patients did not immediately remember exacerbations and described them as "forgotten events". Furthermore, we identified the heterogeneity of exacerbations as an important barrier that differentiated patients recognizing exacerbations late from patients early recognizing exacerbations. However, previous studies have reported that most patients had predictable symptoms and could easily identify consistent warning signs, which may have been due to the inclusion of patients with more exacerbation experiences in those studies. ${ }^{22,24}$ Moreover, we found that recognition was complicated by patients' habituation to their stable symptom severity and daily fluctuations, which has not been described in previous research to our knowledge.

An important strength of this study was that data were independently analyzed by two researchers and discussed with an expert on qualitative research during the entire process. Furthermore, both credibility and confirmability were enhanced by systematically discussing the interpretation of data with experts in the field. Although the results of this study were based on fifteen patients, maximum variation sampling increased the likelihood of diversity in our data collected from a representative selection of patients, which contributed to the transferability of the study. However, this study had a few limitations. Medical chart reviews showed afterward that the inclusion and exclusion criteria were not fully met in two patients with GOLD stage 1. Their charts showed conflicting data with regard to lung function and GOLD stage, and one patient had experienced an exacerbation $>12$ months ago. Nonetheless, these patients were not excluded from the study since they were considered to be eligible by their HCPs and were able to share their experiences regarding exacerbations. Furthermore, the interpretation of data was based on patients' perceptions regarding exacerbations. This might have affected the credibility of our study since previous research has shown that some patients show little understanding regarding exacerbations. ${ }^{21,22}$ To address this potential limitation, we clarified the term exacerbation by asking patients specifically if they could remember a period of symptom deterioration for which medical treatment was indicated.
The conceptual model provided insight into different patterns of exacerbation-related self-management and factors influencing this behavior. Since needs and requests for care flowed naturally from the problems perceived by patients, ${ }^{19}$ the identified influencing factors guided our implications for practice and future research. The conceptual model provides a framework for HCPs to identify patterns in patients' exacerbation-related self-management and stresses the need for better assessment on influencing factors to enhance selfmanagement by eliminating identified barriers. When an HCP identifies poor exacerbation-related self-management, self-management support should be intensified, which may consist of teaching self-monitoring skills and exacerbation education supported by an action plan. ${ }^{16,44}$ HCPs should be aware that these patients might express limited needs regarding self-management support themselves. Patients who recognize exacerbations, and show adequate selfmanagement skills, may benefit from continued education and decision support. HCPs should notice that patients who postpone health care contact might be experiencing barriers in the patient-HCP relationship. For this subgroup of patients, it is important that they are being heard by their HCP and that HCPs listen carefully and take their patient' expertise seriously. Finally, patients who show adequate exacerbationrelated self-management may benefit from continued selfmanagement support and possibly self-treatment.

Moreover, the conceptual model is important for future development of self-management interventions since it shows which influencing factors should be taken into account during the development and delivery of interventions. Based on this model, special attention should be paid to the elimination of barriers in the process of self-management to be able to develop effective self-management interventions that fit patients' perceptions, capabilities, needs and request for care. Future research should focus on the development of targeted and tailored interventions to improve the full spectrum of exacerbation-related self-management and, subsequently, reduce the impact of exacerbations.

\section{Conclusion}

This study provided in-depth insight into factors influencing exacerbation-related self-management in COPD patients. Several patterns in exacerbation-related selfmanagement behavior were identified based on two important self-management skills: recognition of an exacerbation and performance of self-management actions. This study confirmed that recognition of exacerbations can be challenging and that self-management actions are often inadequate. 
The conceptual model explaining factors that influence exacerbation-related self-management can be used as a framework for health care professionals providing selfmanagement support and as a guide for the development of future individualized and tailored exacerbation-related self-management interventions.

\section{Acknowledgments}

We thank all patients who participated in the interviews for sharing their time and experiences, and we acknowledge all health care centers for recruiting patients for this study. Furthermore, we thank Marlies Schrijvers, PhD, for her peer review of this study's methodology and Remco Verbrugge, $\mathrm{MSc}$, for his peer review of the conceptual model through his expertise as a COPD clinical nurse specialist. No funding was provided for this study.

\section{Disclosure}

The authors report no conflicts of interest in this work.

\section{References}

1. Global Initiative for Chronic Obstructive Lung Disease (GOLD). Global Strategy for the Diagnosis, Management, and Prevention of Chronic Obstructive Pulmonary Disease [updated 2016]. Available from: http:// goldcopd.org/global-strategy-diagnosis-management-prevention-copd2016/. Accessed February 2, 2016.

2. Rodriguez-Roisin R. Toward a consensus definition for COPD exacerbations. Chest. 2000;117(5 suppl 2):398S-401S.

3. Burge S, Wedzicha JA. COPD exacerbations: definitions and classifications. Eur Respir J Suppl. 2003;41:46s-53s.

4. Miravitlles M, Ferrer M, Pont A, et al; IMPAC Study Group. Effect of exacerbations on quality of life in patients with chronic obstructive pulmonary disease: a 2 year follow up study. Thorax. 2004;59(5):387-395.

5. Seemungal TA, Donaldson GC, Paul EA, Bestall JC, Jeffries DJ, Wedzicha JA. Effect of exacerbation on quality of life in patients with chronic obstructive pulmonary disease. Am J Respir Crit Care Med. 1998; 157(5 pt 1):1418-1422.

6. Donaldson GC, Seemungal TA, Bhowmik A, Wedzicha JA. Relationship between exacerbation frequency and lung function decline in chronic obstructive pulmonary disease. Thorax. 2002;57(10):847-852.

7. Soler-Cataluna JJ, Martinez-Garcia MA, Roman Sanchez P, Salcedo E, Navarro M, Ochando R. Severe acute exacerbations and mortality in patients with chronic obstructive pulmonary disease. Thorax. 2005; 60(11):925-931.

8. Bourbeau J, van der Palen J. Promoting effective self-management programmes to improve COPD. Eur Respir J. 2009;33(3):461-463.

9. Effing TW, Vercoulen JH, Bourbeau J, et al. Definition of a COPD self-management intervention: International expert group consensus. Eur Respir J. 2016;48(1):46-54.

10. Wilkinson TM, Donaldson GC, Hurst JR, Seemungal TA, Wedzicha JA. Early therapy improves outcomes of exacerbations of chronic obstructive pulmonary disease. Am J Respir Crit Care Med. 2004;169(12): 1298-1303.

11. Bucknall CE, Miller G, Lloyd SM, et al. Glasgow supported selfmanagement trial (GSuST) for patients with moderate to severe COPD: randomised controlled trial. BMJ. 2012;344:e1060.

12. Trappenburg JC, Schaap D, Monninkhof EM, et al. How do COPD patients respond to exacerbations? BMC Pulm Med. 2011;11:43.
13. Bischoff EW, Hamd DH, Sedeno M, et al. Effects of written action plan adherence on COPD exacerbation recovery. Thorax. 2011;66(1): 26-31.

14. Xu W, Collet JP, Shapiro S, et al. Negative impacts of unreported COPD exacerbations on health-related quality of life at 1 year. Eur Respir $J$. 2010;35(5):1022-1030.

15. Langsetmo L, Platt RW, Ernst P, Bourbeau J. Underreporting exacerbation of chronic obstructive pulmonary disease in a longitudinal cohort. Am J Respir Crit Care Med. 2008;177(4):396-401.

16. Trappenburg JC, Monninkhof EM, Bourbeau J, et al. Effect of an action plan with ongoing support by a case manager on exacerbation-related outcome in patients with COPD: a multicentre randomised controlled trial. Thorax. 2011;66(11):977-984.

17. Trappenburg J, Jonkman N, Jaarsma T, et al. Self-management: one size does not fit all. Patient Educ Couns. 2013;92(1):134-137.

18. Spruit MA, Franssen FM, Rutten EP, Wopereis S, Wouters EF, Vanfleteren LE. A new perspective on COPD exacerbations: Monitoring impact by measuring physical, psychological and social resilience. Eur Respir J. 2016;47(4):1024-1027.

19. van Meijel B, Gamel C, van Swieten-Duijfjes B, Grypdonck MH. The development of evidence-based nursing interventions: methodological considerations. J Adv Nurs. 2004;48(1):84-92.

20. Barnes N, Calverley PM, Kaplan A, Rabe KF. Chronic obstructive pulmonary disease and exacerbations: patient insights from the global hidden depths of COPD survey. BMC Pulm Med. 2013;13:54.

21. Adams R, Chavannes N, Jones K, Ostergaard MS, Price D. Exacerbations of chronic obstructive pulmonary disease - a patients' perspective. Prim Care Respir J. 2006;15(2):102-109.

22. Kessler R, Stahl E, Vogelmeier C, et al. Patient understanding, detection, and experience of COPD exacerbations: an observational, interviewbased study. Chest. 2006;130(1):133-142.

23. Harrison SL, Apps L, Singh SJ, Steiner MC, Morgan MD, Robertson N. 'Consumed by breathing' - a critical interpretive meta-synthesis of the qualitative literature. Chronic Illn. 2014;10(1):31-49.

24. Williams V, Hardinge M, Ryan S, Farmer A. Patients' experience of identifying and managing exacerbations in COPD: a qualitative study. NPJ Prim Care Respir Med. 2014;24:14062.

25. Glaser BG, Strauss AL. The Discovery of Grounded Theory: Strategies for Qualitative Research. 7th ed. Elmer, NJ: Aldine; 1967.

26. Boeije H. Analysis in Qualitative Research. 1st ed. London: Sage Publications; 2010

27. Strauss AL, Corbin J. Basics of Qualitative Research. Techniques and Procedures for Developing Grounded Theory. 3rd ed. Thousand Oaks, CA: Sage Publications; 2007.

28. Wester F. Strategieën voor kwalitatief onderzoek. [Strategies for Qualitative Research]. 3rd ed. Muiderberg: Coutinho; 1995.

29. Lincoln YS, Guba EG. Naturalistic Inquiry. Newbury Park, CA: Sage Publications; 1985

30. Hernandez P, Balter M, Bourbeau J, Hodder R. Living with chronic obstructive pulmonary disease: a survey of patients' knowledge and attitudes. Respir Med. 2009;103(7):1004-1012.

31. Rosenstock IM, Strecher VJ, Becker MH. Social learning theory and the health belief model. Health Educ Q. 1988;15(2):175-183.

32. Bandura A. Self-efficacy: toward a unifying theory of behavioral change. Psychol Rev. 1977;84(2):191-215.

33. Bandura A. Self-efficacy. In: Ramachaudran VS, editor. Encyclopedia of Human Behavior. Vol. 4. New York, NY: Academic Press; 1994:71-81

34. Vercoulen JH. A simple method to enable patient-tailored treatment and to motivate the patient to change behaviour. Chron Respir Dis. 2012; 9(4):259-268.

35. Boer LM, Daudey L, Peters JB, Molema J, Prins JB, Vercoulen $\mathrm{JH}$. Assessing the stages of the grieving process in chronic obstructive pulmonary disease (COPD): validation of the acceptance of disease and impairments questionnaire (ADIQ). Int J Behav Med. 2014;21(3):561-570. 
36. Cicutto L, Brooks D, Henderson K. Self-care issues from the perspective of individuals with chronic obstructive pulmonary disease. Patient Educ Couns. 2004;55(2):168-176.

37. Disler RT, Gallagher RD, Davidson PM. Factors influencing selfmanagement in chronic obstructive pulmonary disease: an integrative review. Int J Nurs Stud. 2012;49(2):230-242.

38. Dowson CA, Town GI, Frampton C, Mulder RT. Psychopathology and illness beliefs influence COPD self-management. J Psychosom Res. 2004;56(3):333-340.

39. Bourbeau J. Clinical decision processes and patient engagement in selfmanagement. Dis Manage Health Outcomes. 2008;16(5):327-333.

40. Glanz K, Rimer B, Viswanath K. Theory of reasoned action, theory of planned behaviour, and the integrated behavioral model. Health Behaviour and Health Education. Theory, Research and Practice. 4th ed. San Fransisco, CA: Yossey-Bass; 2008:67-96. Available from: http:// www.sanjeshp.ir/phd/phd_91/Pages/Refrences/health\%20education $\% 20$ and\%20promotion/[Karen_Glanz,_Barbara_K._Rimer,_K._Viswanath] Heal(BookFi.or.pdf. Accessed February 2, 2016.
41. Gruffydd-Jones K, Langley-Johnson C, Dyer C, Badlan K, Ward S What are the needs of patients following discharge from hospital after an acute exacerbation of chronic obstructive pulmonary disease (COPD)? Prim Care Respir J. 2007;16(6):363-368.

42. Korpershoek Y, Bos-Touwen ID, de Man-van Ginkel JM, Lammers JW, Schuurmans MJ, Trappenburg J. Determinants of activation for selfmanagement in patients with COPD. Int J Chron Obstruct Pulmon Dis. 2016;11:1757-1766.

43. Bos-Touwen I, Schuurmans M, Monninkhof EM, et al. Patient and disease characteristics associated with activation for self-management in patients with diabetes, chronic obstructive pulmonary disease, chronic heart failure and chronic renal disease: A cross-sectional survey study. PLoS One. 2015;10(5):e0126400.

44. Zwerink M, Brusse-Keizer M, van der Valk PD, et al. Self management for patients with chronic obstructive pulmonary disease. Cochrane Database Syst Rev. 2014;(3):CD002990. 


\section{Supplementary material}

Table SI Topic list

\begin{tabular}{|c|c|c|}
\hline Topics & Specifications & Questions (examples) \\
\hline \multirow[t]{3}{*}{$\begin{array}{l}\text { Perceived symptoms } \\
\text { of COPD }\end{array}$} & Experience of COPD in daily life & $\begin{array}{l}\text { Standard opening question: How do you experience having a lung disease in your } \\
\text { daily life? }\end{array}$ \\
\hline & Course of the condition & Could you tell me about the course of your condition so far? \\
\hline & Day-to-day variations in symptoms & How do you experience day-to-day variations in symptoms? \\
\hline \multirow[t]{6}{*}{$\begin{array}{l}\text { Perceptions toward } \\
\text { exacerbations }\end{array}$} & Recall of an exacerbation & $\begin{array}{l}\text { Can you remember a period of increased symptoms (for which prednisolone and/or } \\
\text { antibiotics were indicated) and can you describe your experience of this period? }\end{array}$ \\
\hline & Definition of an exacerbation & $\begin{array}{l}\text { Which name does your health care professional give to this period of increased } \\
\text { symptoms? }\end{array}$ \\
\hline & Experience of an exacerbation & How do you experience this period of increased symptoms? \\
\hline & Recognition & $\begin{array}{l}\text { How do you recognize feeling worse than normal? Which symptoms do you } \\
\text { experience indicating the start of an exacerbation? }\end{array}$ \\
\hline & Taking action & What do you do when you experience an increase in symptoms? Why? \\
\hline & \multicolumn{2}{|c|}{$\begin{array}{l}\text { I would like to talk with you about the specific period of increased symptoms. Please remember a recent period of an } \\
\text { exacerbation. }\end{array}$} \\
\hline During an & Performed actions & Which actions did you perform to feel better? Why? \\
\hline \multirow[t]{2}{*}{ exacerbation } & Wish for change & $\begin{array}{l}\text { When you remember this period, are there any actions that you would change in } \\
\text { case of a future exacerbation? }\end{array}$ \\
\hline & Patients' needs & Who, or what, could help you to perform certain actions at a future exacerbation? \\
\hline Perceptions toward & Cause of an exacerbation & What do you think that causes an exacerbation? \\
\hline \multirow[t]{2}{*}{ own role } & $\begin{array}{l}\text { Own influence on exacerbation } \\
\text { course }\end{array}$ & Do you think you can influence the course of an exacerbation yourself? And how? \\
\hline & Importance of own actions & $\begin{array}{l}\text { What is in your opinion the importance of early recognition of an exacerbation and } \\
\text { taking actions yourself? }\end{array}$ \\
\hline $\begin{array}{l}\text { After an } \\
\text { exacerbation }\end{array}$ & Performed actions & $\begin{array}{l}\text { Which actions did you perform after an exacerbation? Why? What was important } \\
\text { for you in this period? }\end{array}$ \\
\hline \multirow[t]{2}{*}{$\begin{array}{l}\text { Self-management in } \\
\text { stable phase }\end{array}$} & Performed actions & $\begin{array}{l}\text { When you feel relatively stable over a period, which actions do you perform to } \\
\text { manage your condition? Why? }\end{array}$ \\
\hline & $\begin{array}{l}\text { Confidence in self-management } \\
\text { toward future exacerbations }\end{array}$ & Do you feel confident in managing a future exacerbation? \\
\hline $\begin{array}{l}\text { Additional questions } \\
\text { regarding previous }\end{array}$ & $\begin{array}{l}\text { Advice from health care } \\
\text { professional }\end{array}$ & Which advice did you receive from your health care professional? \\
\hline topics & & Do you have anything to add to the questions I have asked? \\
\hline
\end{tabular}

\section{Publish your work in this journal}

The International Journal of COPD is an international, peer-reviewed journal of therapeutics and pharmacology focusing on concise rapid reporting of clinical studies and reviews in COPD. Special focus is given to the pathophysiological processes underlying the disease, intervention programs, patient focused education, and self management protocols.

\section{Dovepress}

This journal is indexed on PubMed Central, MedLine and CAS. The manuscript management system is completely online and includes a very quick and fair peer-review system, which is all easy to use. Visit $\mathrm{http}: / /$ www.dovepress.com/testimonials.php to read real quotes from published authors. 